\title{
Reflectivity of metallodielectric photonic glasses
}

\author{
Krassimir P. Velikov, ${ }^{1, *}$ Willem L. Vos, ${ }^{2}$ Alexander Moroz, ${ }^{1, \dagger}$ and Alfons van Blaaderen ${ }^{1, *}$ \\ ${ }^{1}$ Soft Condensed Matter, Debye Institute, Utrecht University, Princetonlaan 5, 3584 CC Utrecht, The Netherlands \\ ${ }^{2}$ Department of Science and Technology and MESA + Research Institute, University of Twente, \\ P.O. Box 217, 7500 AE Enschede, The Netherlands
}

(Received 23 September 2003; published 20 February 2004)

\begin{abstract}
We report on the fabrication and optical properties of metallodielectric photonic glasses of colloidal silver spheres with a radius ranging from 200 to $420 \mathrm{~nm}$ and volume fractions around $60 \%$. Strong modulations $(\sim 25 \%)$ in the optical reflectivity were observed in the visible range for these structures, despite the presence of only short-range order. Despite the strong wavelength dependence of silver dielectric constant, the spectral positions of the optical reflection peaks, with the exception of the smallest size $(200 \mathrm{~nm})$, scale linearly with the particle radius. For the smallest particle size studied, a peak at longer wavelengths related to a lower order gap was observed. Remarkably, the general features in the reflectivity spectra have a qualitative similarity to spectra calculated for light incident on (111) oriented face-centered-cubic (fcc) crystals.
\end{abstract}

DOI: 10.1103/PhysRevB.69.075108

PACS number(s): 42.70.Qs, 82.70.Dd, 42.25.Dd, 81.05.Zx

\section{INTRODUCTION}

Photonic crystals (PCs), materials with a periodic dielectric permittivity with spacings on the order of the wavelength of light, have received a great deal of attention due to their potential in controlling the propagation and spontaneous emission of light. ${ }^{1}$ When light travels in a photonic crystal, it can be diffracted by the periodic dielectric constant, leading to the formation of stop gaps. This interaction is analogous to how the flow of electrons is influenced by the positively charged atoms in a metal or semiconductor crystal lattice. A photonic band gap (PBG) can be formed if the stop gaps overlap for all directions of propagation and for all polarizations. Metallodielectric PCs have been proposed recently as a possible candidate for achieving a PBG in the optical or visible wavelength range. ${ }^{2,3}$ Theoretical studies have shown that a PBG can form in crystals with many different symmetries. ${ }^{3}$ In addition, the gap appears to be more robust against disorder than for purely dielectric PCs.

Disorder in the form of defects and polydispersity in size is always present, both in PCs fabricated using self-assembly methods, ${ }^{4,5}$ and in lithographically defined crystals. ${ }^{6}$ Disorder was found to play a critical role in determining the photonic properties of these structures. ${ }^{4,6}$ For instance, calculations forecast that the PBG in an air-sphere crystal is strongly affected and, in some cases, can be closed by the size polydispersity or the displacements of the spheres used in the initial template. ${ }^{7}$ Stacking faults in crystals, another common type of disorder, were predicted to introduce localized states within the PBG. Indeed, evidence of an Anderson localization of light has been found in completely disordered photonic systems. ${ }^{8}$ On the other hand, amorphous semiconductors, like silicon, do possess an electronic band gap despite the absence of long-range order. Similarly, in two-dimensional (2D) PCs of purely dielectric scatterers, the lowest lying gap survived even in the case of only short-range ordered glassy structures. ${ }^{9}$ It is interesting to see to what extent this also holds for 3D metallodielectric colloidal glasses. Colloidal glasses have short-range order and have been studied exten- sively as a model system for the glass transition. ${ }^{10}$ On the other hand, the photonic properties of colloidal glasses have hardly been studied. ${ }^{11}$ This is despite the fact that it was suggested earlier that randomly distributed metal particles in a dielectric host may be a suitable system to study light localization. ${ }^{12}$ The experimental study of metallodielectric systems with short-range order will give an important contribution toward understanding how photonic properties of crystals are affected by disorder. Finally, from a practical point of view, it is easier and cheaper to fabricate amorphous glasslike photonic materials than crystals. For example, recently demonstrated metallic PCs made of tungsten with a PBG in the infrared may find use in thermophotovoltaic applications, but would be much cheaper if the band gap is present in a glassy state as well. ${ }^{13}$

In this paper, we report on the fabrication and optical properties of photonic glasses of polydisperse silver $(\mathrm{Ag})$ colloidal particles dispersed in water. The polydispersity in the sphere sizes prevented crystallization at a high volume fraction. ${ }^{14}$ Optical reflectivity measurements were performed to quantify the modulations in the reflectivity. To investigate the similarities between metallodielectric glasses and crystals, theoretical reflectivity spectra of face-centered-cubic (fcc) crystals were calculated for comparison.

\section{EXPERIMENT}

The synthesis and optical characterization of the silver colloidal particles, which are spherical aggregates of small Ag nanoparticles, are described elsewhere. ${ }^{14}$ The effective dielectric constant of individual $\mathrm{Ag}$ spheres was modeled using the Bruggeman effective medium approach in which randomly connected bulk Ag immersed in water is assumed. ${ }^{14}$ An experimentally determined wavelengthdependent dielectric constant for bulk $\mathrm{Ag}$ was used, ${ }^{15}$ whereas the wavelength dependence of the dielectric constant of water was neglected. A filling fraction 0.80 of bulk silver in a single particle was determined from extinction spectra measurements. ${ }^{14}$ Colloidal glasses were made from 


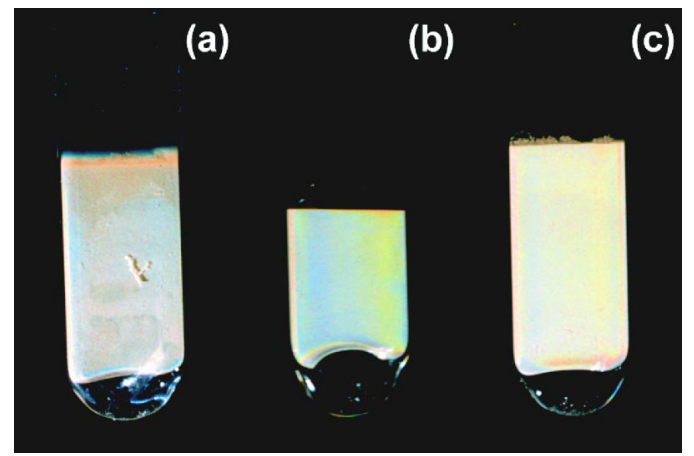

FIG. 1. (Color online) Optical photographs of capillaries filled with sedimented silver particles dispersed in water with different mean radii $(R)$ : (a) $R=197 \mathrm{~nm}$, (b) $R=349 \mathrm{~nm}$, and (c) $R$ $=418 \mathrm{~nm}$. All samples display bright reflection colors under illumination with white light.

particles with different mean radius $R$ between 200 and 420 $\mathrm{nm}$ and size polydispersity (the relative width of the first moment of the Gaussian distribution in particles sizes) $\delta$ $<15 \%$. In some cases, these particles formed chargestabilized crystals with low $(<50 \%)$ volume fraction, but these were not studied in the present paper. ${ }^{14}$ Samples for reflectivity measurements were prepared by filling thin $(0.20$ $\mathrm{mm})$ glass capillaries (VitroCom) with a colloidal suspension, allowing the particles to sediment under gravity. To examine the microscopic structure, we used a confocal microscope. Samples in water were observed in reflection mode $(\lambda=632 \mathrm{~nm})$ with an inverted confocal microscope (Leica DM IRB, in combination with a Leica TCS NT scanhead) with an oil-immersion lens (Leica, 100×, NA 1.4). Vertically standing capillaries were examined with the microscope tilted at $90^{\circ}$. The confocal images were analyzed using IDL (Research Systems, Inc.) routines to filter, locate particle coordinates and calculate the radial distribution function $g(r)$.

The setup for the frequency-resolved reflectivity measurements is similar to that described in Ref. 16. The output of a broadband xenon lamp is focused on a pinhole, which acts as a point source. The light is collimated and sent through a Fourier-transform interferometer (Biorad FTS-60A) operating with a spectral resolution of $16 \mathrm{~cm}^{-1}$. The light beam is focused to a 0.5 -mm-diameter spot on the sample. The samples in a capillary were mounted vertically on a goniometer head to align the face of the capillary perpendicularly to the incident beam. The reflected light is collected by a $\mathrm{Si}$ diode, which yields an interferogram that is Fourier transformed to get the spectrum of the reflected light. The spectra were referenced to a silver mirror with $>95 \%$ reflectivity.

\section{RESULTS AND DISCUSSION}

Figure 1 shows optical photographs of capillaries filled with silver particles of different sizes sedimented in water. Under illumination with white light, these samples display bright colors at almost all angles of observation. The structure of the particles close to the glass wall was studied by reflection confocal microscopy. Due to the negative net

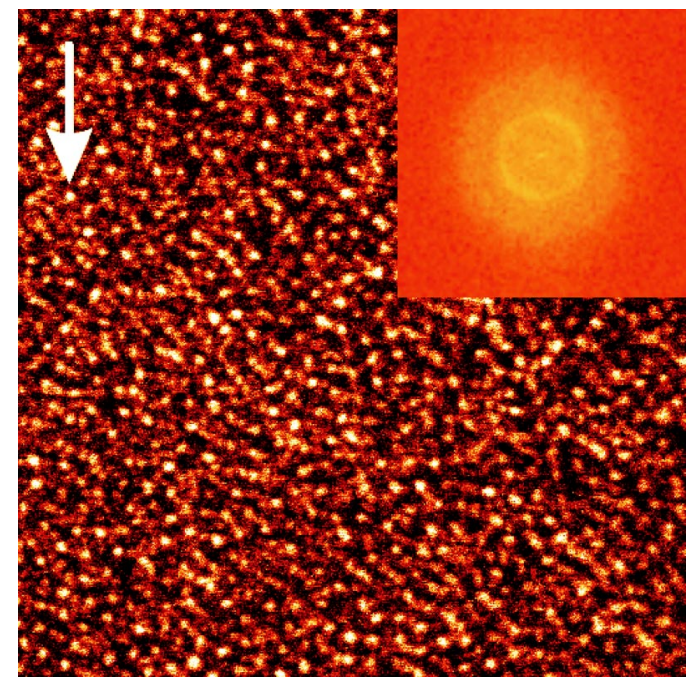

FIG. 2. (Color online) Typical reflection confocal image (25 $\left.\times 25 \mu \mathrm{m}^{2}\right)$ of a photonic glass of $\mathrm{Ag}$ particles $(R=418 \mathrm{~nm})$ dispersed in water next to the wall of the capillary. The inset shows a Fourier transform of the image. The mean interparticle distance determined after determining the particle coordinates is $A$ $=874 \mathrm{~nm}$ which correspond to a volume fraction $\phi=0.65$. The arrow points in the direction of gravity.

charge, the particles do not stick to the glass wall and could be redispersed as individual spheres. ${ }^{14}$ Figure 2 shows a typical confocal image of a charged-stabilized colloidal glass. Because of the strong interaction of the silver particles with light, particles that are deeper inside the sample could not be imaged, as the point spread function of the microscope is already completely scrambled after the first few layers. Nevertheless, in analogy to hard-sphere and charge-stabilized colloidal glasses of (fluorescent) dielectric particles studied by confocal microscopy, we can assume that the glassy structure extends throughout the whole sample, as a flat wall is known to increase order compared to the bulk structure. ${ }^{17}$ The brightest spots in the image (Fig. 2) correspond to the particles that are closest to the glass wall. They were used to determine the volume fraction. All samples had a typical amorphous glasslike structure, as expected for particles with polydispersity larger than $\sim 7 \% .{ }^{14}$ The ringlike patterns in the Fourier transform image, shown in the inset, also indicate the presence of short-range order only. Confocal images were taken at different heights in the sediments in the capillaries. Unfortunately, because of the fact that we can image only the particles closest to the wall and the fact that these particles are not arranged in perfect layers, the mean interparticle distance $A$ could not be obtained accurately in all cases. For large particles $(R=418 \mathrm{~nm}, \delta=12 \%), A$ was determined from the first peak in $g(r)$ assuming an fcc crystal structure. For the sample shown in Fig. 2, a particle volume fraction of $65 \%$ was obtained. This value is close to that of random close-packed structures of spheres $(63 \%)$. The high polydispersity of the silver particles could additionally increase the volume fraction. ${ }^{18}$ On the other hand, strong electrostatic repulsion between charged silver particles will decrease the effective polydispersity. For smaller particle sizes, the confocal image could not provide a high enough resolu- 

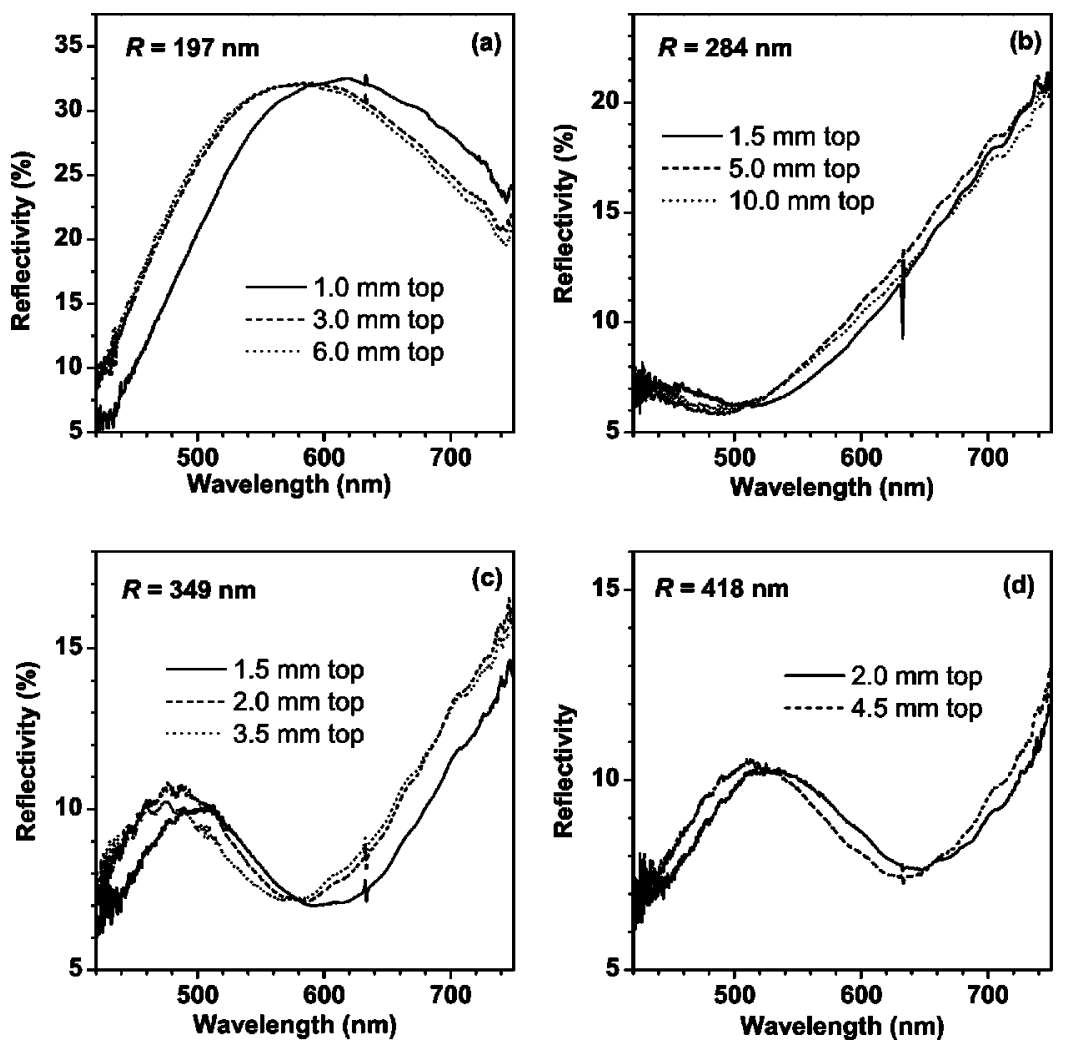

FIG. 3. Experimental specular optical reflectivity at normal incidence as a function of wavelength of the samples taken at different height of the sediment with full height $h$ in capillaries containing particles with different $\operatorname{radii}(R)$ : (a) $R$ $=197 \mathrm{~nm}, \quad h=7 \mathrm{~mm} ; \quad$ (b) $R=284 \mathrm{~nm}, \quad h$ $=15 \mathrm{~mm}$; (c) $R=349 \mathrm{~nm}, h=5 \mathrm{~mm}$; and (d) $R$ $=418 \mathrm{~nm}, h=8 \mathrm{~mm}$. Different curves correspond to measurements at different distances from the top of the sample, giving an indication of changes as a function of the volume fraction. tion to make a reliable coordinate determination. Volume fractions on the order of 55-65\% were estimated from the confocal images. It is important to note here that for all samples the metal spheres were not in conducting contact with each other, contrary to recently prepared "inverse" photonic metallic crystals. ${ }^{19}$

Optical reflectivity spectra measured at normal incidence on photonic glasses of particles of different mean radii are shown in Fig. 3. A strong modulation $(\sim 25 \%)$ in the reflectivity appears in a range of wavelengths in the visible. Broad peaks are observed, likely because of the absence of longrange order. The reflectivity shows a slight dependence on the particle volume fraction $\phi$, which varies along the height of the sample, similarly as observed with dielectric particles. ${ }^{20}$ Except for $R=284 \mathrm{~nm}$ [Fig. 3(b)], all samples display a well-developed and broad maximum in the optical

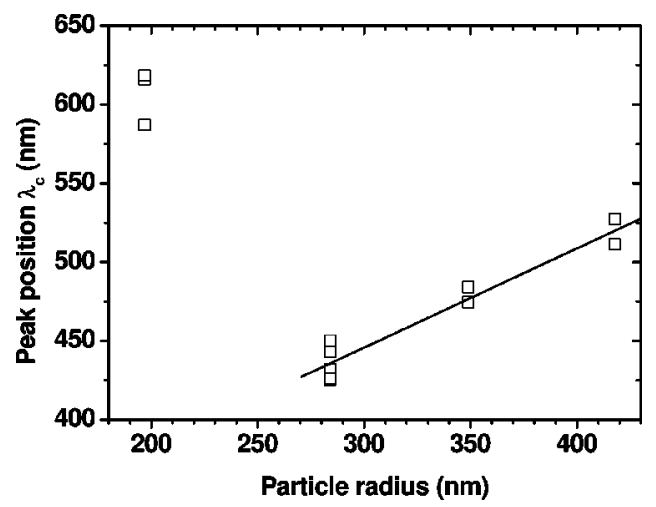

FIG. 4. Positions of the stop gaps that appear in the visible as a function of the particles radius. The line is guide to the eye. reflectivity. All samples, except for the smallest size, display an increase of the reflectivity at long wavelengths. For $R$ $=349$ and $418 \mathrm{~nm}$ a smaller peak appears, which shifts towards longer $\lambda$ with increasing radius $R$. Figure 4 shows the positions of the reflectivity peaks as a function of the particle radius. Despite the strong wavelength dependence of the dielectric constant of silver, the position of the reflection peaks linearly depends on the mean particle radius. The reflectivity maximum for particles of $197 \mathrm{~nm}$ is not in line with the other sizes, as it should appear in the ultraviolet range. Instead, a peak associated with a possible lower order gap is observed in the optical range. We want to emphasize that features in the reflectivity spectra do not resemble the backscattering spectrum of the single particles. The spectra also differ strongly from effective medium calculations, in which we approximate the colloidal glass as a slab of metallodielectric material with an effective refractive index including the metallic dispersion. ${ }^{21}$ Because it is known that concentrated colloidal liquids and glasses at a smooth wall show layering perpendicular to the wall with a local sixfold symmetry of the particles arranged in each layer, we compared the experimental reflectivity curves with calculated curves of (111) oriented fcc crystals.

We performed calculations for six layers using the layer Korringa-Kohn-Rostoker (KKR) method. ${ }^{22}$ In our earlier study on purely dielectric PCs, the method reproduced the experimental spectra satisfactorily. ${ }^{23}$ Six layers were found to be sufficient to consider the crystal being infinitely thick for all particle sizes and $\phi$ 's studied. The calculations showed that for $R>284 \mathrm{~nm}$ even three layers were already similar to the spectra of a thick crystal. The model crystal was constructed from a dielectric slab (capillary glass wall of 

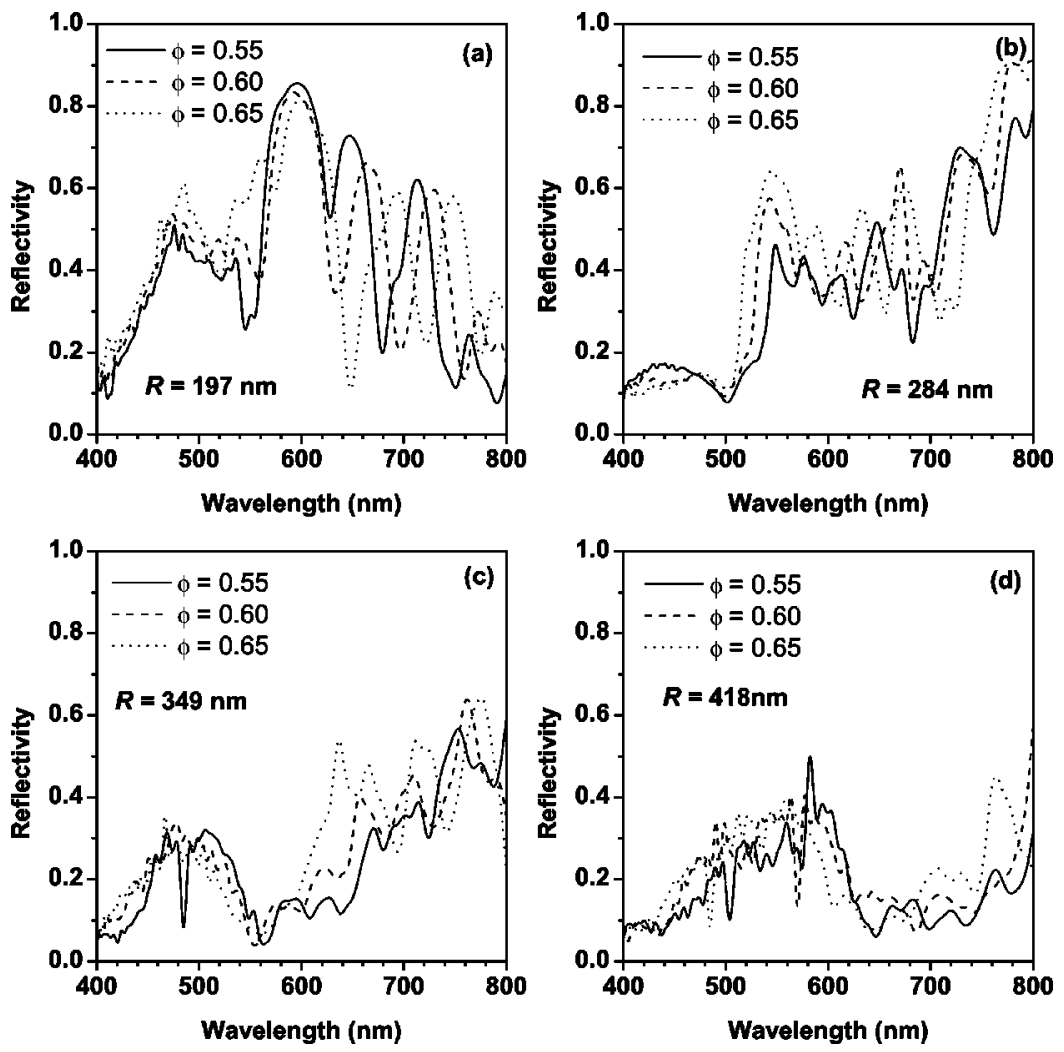

FIG. 5. Theoretical reflectivity spectra along the (111) crystallographic axis as a function of the wavelength for a six-layer fcc crystal made of particles of different radii $(R)$ chosen to match the measured samples shown in Fig. 3: (a) $R$ $=200 \mathrm{~nm}$, (b) $R=285 \mathrm{~nm}$, (c) $R=350 \mathrm{~nm}$, and (d) $R=420 \mathrm{~nm}$. The spectra were calculated for crystals with different volume fraction $(\phi)$. For clarity, all spectra were smoothed using a Fourier filter $\left(\sim 0.1 \mathrm{~nm}^{-1}\right)$. thickness $\left.200 \mu \mathrm{m}, n_{s}=1.450\right)$ and six layers of Ag spheres immersed in water acting as host $\left(n_{h}=1.330\right)$. The resulting spectra display many narrow resonances due to interference between the dielectric slab and crystal interfaces. For clarity, Fourier smoothing was applied on all spectra. ${ }^{24}$ Figure 5 shows calculated specular reflectivity at normal incidence on the (111)-crystallographic plane of six-layer-thick fcc crystals with different volume fractions and different sphere sizes in water behind a glass wall. We want to emphasize that due to the coupling of evanescent waves on the crystal surface and propagating waves in the dielectric slab, the reflectivity spectra differ from spectra obtained from a free standing crystal. $^{25}$ The resulting theoretical reflectivity spectra (Fig. 5) are not very sensitive to the volume fraction $\phi$. Some general features remain almost unchanged in a large region of $\phi$ $=0.55-0.74$. By comparing Figs. 3 and 5 we find that the main features of the experimental spectra are reproduced by the theoretical calculations of the spectra in the (111) direction, while there was no correspondence with the spectra in the (100) direction (not shown). For example, Fig. 5(a) features a broad maximum centered around $600 \mathrm{~nm}$, which was experimentally observed [Fig. 3(a)]. In Fig. 5(b) we can identify an initial relatively flat region, a minimum around $500 \mathrm{~nm}$, and a subsequent up-turn at longer wavelengths. In Figs. 5(c) and 5(d), we can identify a broad maximum, which was also observed in the amorphous samples [Figs. 3(c) and 3(d)]. The position of the peak roughly scales with $R$ as in the experimental spectra (Fig. 4). At long wavelengths, the behavior also compares well with the experimental spectra.

The origin of the features in the optical spectra is difficult to identify exactly, but they are associated with the higher order stop band in the (111) direction. From the photonic band structure calculations (data not shown), for certain parameters a $\mathrm{PBG}$ is expected in the far infrared region. It is also evident that the observed peaks in the reflectivity do not result from the same order stop band. The strong analogy between reflectivity spectra of ordered and disordered metallodielectric PCs, evident from our results, warrants further research to explore the similarities between amorphous semiconductors and metallodielectric photonic glasses. For instance, it would be very important to investigate both theoretically and experimentally if the strong modulation in the density of states present in the crystal can also be found in the glasses. A PBG in a metallodielectric PC has been shown to persist for a variety of refractive indices of the ambient, which fills the space between metallic particles. ${ }^{2}$

\section{CONCLUSIONS}

In conclusion, we have studied the optical properties of metallodielectric photonic glasses of colloidal silver particles in water. These samples display bright colors, observable by the naked eye, when illuminated with white light. We demonstrated experimentally the presence of a strongly modulated specular reflectivity in the visible for these photonic glasses despite the presence of short-range order only. The general features in the experimental spectra are surprisingly in qualitative agreement with the reflectivity spectra calculated for (111)-oriented fcc crystals. Our findings suggest that some metallophotonic applications might be performed more cheaply and easily with amorphous metallodielectric materials. 


\section{ACKNOWLEDGMENTS}

We are grateful to Gabby E. Zegers (Utrecht University) for the preparation and characterization of the silver colloidal particles, and to Arnout Imhof (Utrecht University) and
Rudolf Sprik (University of Amsterdam) for stimulating discussions. This work was part of the research program of the Stichting voor Fundamenteel Onderzoek der Materie (FOM), which is financially supported by the Nederlandse Organisatie voor Wetenschappelijk Onderzoek (NWO).
*Corresponding author. Present address: Unilever Research, P.O. Box 114, 3130 AC Vlaardingen, The Netherlands. Electronic mail: Krassimir.Velikov@Unilever.com

†Present address: EPO, Gitschiner Strasse 103, 10969 Berlin, Germany.

†Corresponding author. Electronic mail: A.vanBlaaderen @ phys.uu.nl

${ }^{1}$ V. P. Bykov, Sov. J. Quantum Electron. 4, 861 (1975); E. Yablonovitch, Phys. Rev. Lett. 58, 2059 (1987); S. John, Phys. Rev. Lett. 58, 2486 (1987); Photonic Crystals and Light Localization in the 21st Centry, edited by C. M. Soukoulis, NATO Science Series C (Kluwer Academic, Dordrecht, 2001).

${ }^{2}$ A. Moroz, Phys. Rev. Lett. 83, 5274 (1999); Phys. Rev. B 66, 115109 (2002).

${ }^{3}$ W. Y. Zhang, X. Y. Lei, Z. L. Wang, D. G. Zheng, W. Y. Tam, C. T. Chan, and P. Sheng, Phys. Rev. Lett. 84, 2853 (2000).

${ }^{4}$ Y. A. Vlasov, V. N. Astratov, A. V. Baryshev, A. A. Kaplyanskii, O. Z. Karimov, and M. F. Limonov, Phys. Rev. E 61, 5784 (2000); Y. A. Vlasov, M. Deutsch, and D. J. Norris, Appl. Phys. Lett. 76, 1627 (2000); A. F. Koenderink, M. Megens, G. van Soest, W. L. Vos, and A. Lagendijk, Phys. Lett. A 268, 104 (2000).

${ }^{5}$ J. F. Galisteo Lopez and W. L. Vos, Phys. Rev. E 66, 036616 (2002).

${ }^{6}$ M. J. A. de Dood, A. Polman, and J. G. Fleming, Phys. Rev. B 67, 115106 (2003).

${ }^{7}$ Z. Y. Li and Z. Q. Zhang, Phys. Rev. B 62, 1516 (2000); R. Biswas, M. M. Sigalas, G. Subramania, C. M. Soukoulis, and K. M. Ho, ibid. 61, 4549 (2000).

${ }^{8}$ D. S. Wiersma, P. Bartolini, A. Lagendijk, and R. Righini, Nature (London) 390, 671 (1997).

${ }^{9}$ C. J. Jin, X. D. Meng, B. Y. Cheng, Z. L. Li, and D. Z. Zhang, Phys. Rev. B 63, 195107 (2001).

${ }^{10}$ W. K. Kegel and A. van Blaaderen, Science 287, 290 (2000).

${ }^{11}$ J. Ballato, J. Dimaio, A. James, and E. Gulliver, Appl. Phys. Lett. 75, 1497 (1999).
${ }^{12}$ S.-I. Lee, T. W. Noh, K. Cummings, and J. R. Gaines, Phys. Rev. Lett. 55, 1626 (1985); K. Arya, Z. B. Su, and J. L. Birman, ibid. 57, 2725 (1986).

${ }^{13}$ J. G. Fleming, S. Y. Lin, I. El-Kady, R. Biswas, and K. M. Ho, Nature (London) 417, 52 (2002).

${ }^{14}$ K. P. Velikov, G. E. Zegers, and A. van Blaaderen, Langmuir 19, 1384 (2003).

${ }^{15}$ Handbook of Optical Constants of Solids, edited by E. D. Palik (Academic, New York, 1985).

${ }^{16}$ M. S. Thijssen, R. Sprik, J. E. G. J. Wijnhoven, M. Megens, T. Narayanan, A. Lagendijk, and W. L. Vos, Phys. Rev. Lett. 83, 2730 (1999).

${ }^{17}$ D. H. van Winkle and C. A. Murray, J. Chem. Phys. 89, 3885 (1988); J. P. Hoogenboom, D. Derks, P. Vergeer, and A. van Blaaderen, ibid. 117, 11320 (2002).

${ }^{18}$ W. Schaertl and H. Sillescu, J. Stat. Phys. 77, 1007 (1994).

${ }^{19}$ O. D. Velev, P. M. Tessier, A. M. Lenhoff, and E. W. Kaler, Nature (London) 401, 548 (1999); N. Eradat, J. D. Huang, Z. V. Vardeny, A. A. Zakhidov, I. Khayrullin, I. Udod, and R. H. Baughman, Synth. Met. 116, 501 (2001).

${ }^{20}$ W. L. Vos, M. Megens, C. M. van Kats, and P. Bosecke, J. Phys.: Condens. Matter 8, 9503 (1996).

${ }^{21}$ The colloidal glass was considered as a metallodielectric slab of silver particles in water. The effective dielectric constant was calculated using either Maxwell-Garnett or Bruggeman effective medium theory (EMTs) with water as a host. The effective dielectric constant of a single Ag particle was calculated using the Bruggeman EMT with silver as a host.

${ }^{22}$ N. Stefanou, V. Yannopapas, and A. Modinos, Comput. Phys. Commun. 113, 49 (1998).

${ }^{23}$ K. P. Velikov, A. Moroz, and A. van Blaaderen, Appl. Phys. Lett. 80, 49 (2002).

${ }^{24}$ The smoothing is accomplished by removing Fourier components with frequencies higher than $\sim 0.1 \mathrm{~nm}^{-1}$.

${ }^{25}$ Y. Kurokawa, H. Miyazaki, and Y. Jimba, Phys. Rev. B 65, 201102 (2002). 